\title{
MEASUREMENTS OF THE RATE OF TYPE Ia SUPERNOVAE AT REDSHIFT $\lesssim 0.3$ FROM THE SLOAN DIGITAL SKY SURVEY II SUPERNOVA SURVEY
}

\author{
Benjamin Dilday ${ }^{1,2,3}$, Mathew Smith ${ }^{4,5}$, Bruce Bassett $^{4,6}$, Andrew Becker $^{7}$, Ralf BendeR ${ }^{8,9}$, Francisco Castander $^{10}$, \\ David Cinabro $^{11}$, Alexei V. Filippenko ${ }^{12}$, Joshua A. Frieman ${ }^{13}{ }^{14}$, Lluís Galbany ${ }^{15}$, Peter M. Garnavich ${ }^{16}$, \\ Ariel Goobar $^{17,18}$, Ulrich Hopp ${ }^{8,9}$, Yutaka Ihara ${ }^{19}$, SaurabH W. Jha ${ }^{1}$, Richard Kessler ${ }^{3,13}$, Hubert Lampeitl ${ }^{5}$, \\ John Marriner ${ }^{14}$, Ramon Miquel ${ }^{15,20}$, Mercedes Mollá ${ }^{21}$, Robert C. Nichol ${ }^{5}$, Jakob Nordin ${ }^{18}$, Adam G. Riess $^{22,23}$, \\ Masao Sako $^{24}$, Donald P. Schneider ${ }^{25}$, Jesper Sollerman ${ }^{17,26}$ J. Craig Wheeler $^{27}$, Linda Östman $^{18}$, Dmitry Bizyaev $^{28}$, \\ Howard Brewington ${ }^{28}$, Elena Malanushenko ${ }^{28}$, Viktor Malanushenko ${ }^{28}$, Dan Oravetz ${ }^{28}$, Kaike Pan ${ }^{28}$, \\ Audrey Simmons ${ }^{28}$, AND STEPHANIE SNEDDEN ${ }^{28}$ \\ ${ }^{1}$ Department of Physics and Astronomy, Rutgers, the State University of New Jersey, 136 Frelinghuysen Road, Piscataway, NJ 08854, USA; \\ bdilday@physics.rutgers.edu \\ ${ }^{2}$ Department of Physics, University of Chicago, Chicago, IL 60637, USA \\ ${ }^{3}$ Kavli Institute for Cosmological Physics, The University of Chicago, 5640 South Ellis Avenue, Chicago, IL 60637, USA \\ ${ }^{4}$ Department of Mathematics and Applied Mathematics, University of Cape Town, Rondebosch 7701, South Africa \\ ${ }^{5}$ Institute of Cosmology and Gravitation, Mercantile House, Hampshire Terrace, University of Portsmouth, Portsmouth PO1 2EG, UK \\ ${ }^{6}$ South African Astronomical Observatory, P.O. Box 9, Observatory 7935, South Africa \\ ${ }^{7}$ Department of Astronomy, University of Washington, Box 351580, Seattle, WA 98195, USA \\ ${ }^{8}$ Max Planck Institute for Extraterrestrial Physics, D-85748 Garching, Germany \\ ${ }^{9}$ Universitaets-Sternwarte Munich, 1 Scheinerstrasse, D-81679 Munich, Germany \\ ${ }^{10}$ Institut de Ciències de l'Espai (IEEC-CSIC), Barcelona, Spain \\ ${ }^{11}$ Department of Physics and Astronomy, Wayne State University, Detroit, MI 48202, USA \\ ${ }^{12}$ Department of Astronomy, University of California, Berkeley, CA 94720-3411, USA \\ ${ }^{13}$ Department of Astronomy and Astrophysics, The University of Chicago, 5640 South Ellis Avenue, Chicago, IL 60637, USA \\ ${ }^{14}$ Center for Astrophysics, Fermi National Accelerator Laboratory, P.O. Box 500, Batavia, IL 60510, USA \\ ${ }^{15}$ Institut de Física d'Altes Energies, Barcelona, Spain \\ ${ }^{16}$ University of Notre Dame, 225 Nieuwland Science, Notre Dame, IN 46556-5670, USA \\ 17 The Oskar Klein Centre, Department of Astronomy, Albanova, Stockholm University, SE-106 91 Stockholm, Sweden \\ ${ }^{18}$ Department of Physics, Stockholm University, Albanova University Center, S-106 91 Stockholm, Sweden \\ ${ }^{19}$ Institute of Astronomy, Graduate School of Science, University of Tokyo 2-21-1, Osawa, Mitaka, Tokyo 181-0015, Japan \\ ${ }^{20}$ Institució Catalana de Recerca i Estudis Avançats, Barcelona, Spain \\ ${ }^{21}$ Centro de Investigaciones Energéticas, Medioambientales y Tecnológicas, Madrid, Spain \\ 22 Space Telescope Science Institute, 3700 San Martin Drive, Baltimore, MD 21218, USA \\ ${ }^{23}$ Department of Physics and Astronomy, Johns Hopkins University, 3400 North Charles Street, Baltimore, MD 21218, USA \\ ${ }^{24}$ Department of Physics and Astronomy, University of Pennsylvania, 209 South 33rd Street, Philadelphia, PA 19104, USA \\ ${ }^{25}$ Department of Astronomy and Astrophysics, 525 Davey Laboratory, The Pennsylvania State University, University Park, PA 16802, USA \\ ${ }^{26}$ Dark Cosmology Centre, Niels Bohr Institute, University of Copenhagen, Denmark \\ ${ }^{27}$ Department of Astronomy, University of Texas, Austin, TX 78712, USA \\ ${ }_{28}$ Apache Point Observatory, P.O. Box 59, Sunspot, NM 88349, USA \\ Received 2009 August 12; accepted 2010 February 16; published 2010 March 30
}

\begin{abstract}
We present a measurement of the volumetric Type Ia supernova (SN Ia) rate based on data from the Sloan Digital Sky Survey II (SDSS-II) Supernova Survey. The adopted sample of supernovae (SNe) includes 516 SNe Ia at redshift $z \lesssim 0.3$, of which $270(52 \%)$ are spectroscopically identified as SNe Ia. The remaining 246 SNe Ia were identified through their light curves; 113 of these objects have spectroscopic redshifts from spectra of their host galaxy, and 133 have photometric redshifts estimated from the SN light curves. Based on consideration of 87 spectroscopically confirmed non-Ia SNe discovered by the SDSS-II SN Survey, we estimate that $2.04_{-0.95}^{+1.61} \%$ of the photometric SNe Ia may be misidentified. The sample of SNe Ia used in this measurement represents an order of magnitude increase in the statistics for SN Ia rate measurements in the redshift range covered by the SDSS-II Supernova Survey. If we assume an SN Ia rate that is constant at low redshift $(z<0.15)$, then the SN observations can be used to infer a value of the SN rate of $r_{V}=\left(2.69_{-0.30-0.01}^{+0.34+0.21}\right) \times 10^{-5} \mathrm{SNe} \mathrm{yr}^{-1} \mathrm{Mpc}^{-3}\left(H_{0} /\left(70 \mathrm{~km} \mathrm{~s}^{-1} \mathrm{Mpc}^{-1}\right)\right)^{3}$ at a mean redshift of $\sim 0.12$, based on $79 \mathrm{SNe}$ Ia of which 72 are spectroscopically confirmed. However, the large sample of SNe Ia included in this study allows us to place constraints on the redshift dependence of the SN Ia rate based on the SDSS-II Supernova Survey data alone. Fitting a power-law model of the SN rate evolution, $r_{V}(z)=A_{p} \times\left((1+z) /\left(1+z_{0}\right)\right)^{\nu}$,

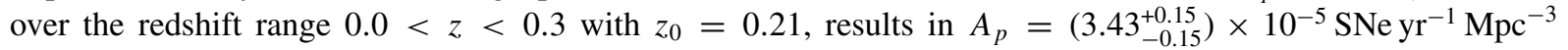
$\left(H_{0} /\left(70 \mathrm{~km} \mathrm{~s}^{-1} \mathrm{Mpc}^{-1}\right)\right)^{3}$ and $v=2.04_{-0.89}^{+0.90}$.
\end{abstract}

Key words: supernovae: general

Online-only material: color figures, machine-readable tables

\section{INTRODUCTION}

Type Ia supernovae ( $\mathrm{SNe}$ Ia) occupy a prominent position in contemporary astrophysics, in part due to their utility as cosmological distance indicators (for a review, see Filippenko 2005). The observed correlation between the peak luminosity and the rate of decline for SNe Ia (Pskovskii 1977; Phillips 1993) has been exploited to improve the accuracy of measured 
distances to $\mathrm{SNe}$ Ia and thereby place important constraints on cosmological models (e.g., Riess et al. 1998, 2004, 2007, 2009; Perlmutter et al. 1999; Astier et al. 2006; Wood-Vasey et al. 2007; Hicken et al. 2009; Freedman et al. 2009; Kessler et al. 2009). However, the SN Ia decline rate versus peak luminosity correlation is mainly an empirically determined phenomenon, and the exact nature of the progenitor systems that give rise to $\mathrm{SNe}$ Ia remains uncertain. A better understanding of $\mathrm{SN}$ Ia progenitor systems is desirable both for investigations of fundamental astrophysics (e.g., binary star evolution and explosion physics) and to provide a theoretical foundation for understanding any possible evolution of SN Ia properties (such as the decline rate versus peak luminosity correlation) with redshift that could cause additional systematic effects in distance measurements.

The SN Ia rate can be used to place important constraints on the progenitor systems of SNe Ia. In general, the $\mathrm{SN}$ Ia rate can be expressed as a delay function convolved with the cosmic star formation rate (SFR; e.g., Greggio 2005). That is,

$$
r(t)=\int_{0}^{t} k_{\Gamma}\left(t^{\prime}\right) \Psi\left(t^{\prime}\right) A_{\mathrm{SN}}\left(t-t^{\prime}\right) D\left(t-t^{\prime}\right) d t^{\prime},
$$

where $r(t)$ is the $\mathrm{SN}$ rate, $\Psi\left(t^{\prime}\right)$ is the SFR, $k_{\Gamma}\left(t^{\prime}\right)$ is the number of stars per unit mass for the population formed at epoch $t^{\prime}$, $A_{\mathrm{SN}}\left(t-t^{\prime}\right)$ is the number of stars from the population that will result in $\mathrm{SN}$ explosions, and $D\left(t-t^{\prime}\right)$ is a distribution of delay times between the formation of a stellar system and the resulting SN explosion. The delay function varies depending on the model assumed for the progenitors of SNe Ia, and measurements of the $\mathrm{SN}$ rate, in combination with measurements of the cosmic SFR, can therefore place observational constraints on SN Ia progenitor models. We emphasize that according to Equation (1), constraints on SN Ia progenitor systems rely not only on precise measurements of the $\mathrm{SN}$ rate, but also on measurements of the cosmic SFR. At present, measurements of the cosmic SFR (as a function of time) suffer from significant uncertainties, thus complicating the interpretation of the cosmic SN rate in terms of delay functions (Förster et al. 2006). In this paper, we focus on presenting our SN rate measurements and will not pursue detailed comparisons to the cosmic SFR.

The SN rate was first measured by Zwicky (1938), who found it to be approximately "one SN per few hundred years per average nebula," in the local universe. Subsequently, improvements in astronomical technology as well as increased observing time dedicated to $\mathrm{SN}$ searches have led to more precise SN rate measurements, spanning a wide range of redshifts. In the local universe, the SN Ia rate has been measured by Cappellaro et al. (1999) from $\sim 140 \mathrm{SNe}$ Ia and by Li et al. (2010a, 2010b) from $\sim 930 \mathrm{SNe}$ Ia. At intermediate redshifts $(0.1 \lesssim z \lesssim 0.5)$, the SN Ia rate has been measured by many authors (e.g., Hardin et al. 2000; Pain et al. 2002; Madgwick et al. 2003; Tonry et al. 2003; Blanc et al. 2004; Neill et al. 2006; Sullivan et al. 2006b; Botticella et al. 2008). At high redshifts, the SN Ia rate has been measured with data from the Hubble Space Telescope (HST) by Dahlen et al. (2004, 2008). All of these SN rate measurements are based on SN Ia samples that are primarily spectroscopically identified and were determined in a manner similar to that of the SDSS-II Supernova Survey SN rate analysis presented here. In addition to these measurements, a number of authors have presented SN Ia rate analyses based on photometric identification of $\mathrm{SNe}$ Ia, in many cases with only a few photometric observations. These include measurement of the intermediateredshift rate by Horesh et al. (2008), the intermediate-to- high-redshift rate by Barris \& Tonry (2006), and the highredshift rate by Poznanski et al. (2007b) and Kuznetsova et al. (2008).

A precise measurement of the low-redshift $(z<0.12) \mathrm{SN}$ Ia rate, based on $17 \mathrm{SNe}$ Ia from the first season of the SDSSII Supernova Survey, was given by Dilday et al. (2008). In this paper we discuss an extension of this volumetric SN Ia rate measurement to a higher redshift limit, based on all three seasons of the SDSS-II Supernova Survey (Frieman et al. 2008). Including $\mathrm{SNe}$ from three years of the SDSS-II Supernova Survey and considering a larger redshift range results in a major increase in the number of SNe used for the rate measurement. At low redshifts, the $\mathrm{SN}$ rate measurements discussed here have the same high purity and completeness as for the low-redshift rate from the first season discussed by Dilday et al. (2008), but with increased statistical power. Inclusion of higher-redshift $\mathrm{SNe}$ allows for investigation of the redshift dependence of the SN Ia rate over the range covered by the SDSS-II Supernova Survey. However, at higher redshifts, systematic uncertainties become increasingly important and eventually dominate the error budget. The efficiency studies and SN selection functions described herein have also been used to estimate the SN Ia rate as an explicit function of the properties of their host galaxies (M. Smith et al. 2010, in preparation) and for studies of the SN Ia rate in clusters of galaxies (Dilday et al. 2010).

The rest of this paper is organized as follows. In Section 2 we briefly describe the observations and SN search strategy of the SDSS-II Supernova Survey. Section 3 discusses selection of the SN rate sample from the SDSS-II Supernova Survey data, and Section 4 determines the efficiency for SN selection. We present our measurement of the SN Ia rate in Section 5, and our conclusions are summarized in Section 6. Whenever necessary, we assume a flat $\Lambda$ CDM universe with $\Omega_{m}=0.3, \Omega_{\Lambda}=0.7$, and $H_{0}=70 \mathrm{~km} \mathrm{~s}^{-1} \mathrm{Mpc}^{-1}$.

\section{SDSS-II SUPERNOVA SURVEY OBSERVATIONS}

Here we briefly describe aspects of the SDSS-II Supernova Survey most relevant to the present SN rate analysis. Much of the material in this section is also relevant to the $\mathrm{SN}$ rate studies described by Dilday et al. (2010), and is discussed more fully therein. The survey is described in more detail by Frieman et al. (2008), and the SN detection algorithms are given by Sako et al. (2008). Additional details of the survey observations and the use of in situ artificial SNe for determining SN detection efficiencies are discussed by Dilday et al. (2008). A technical summary of the Sloan Digital Sky Survey (SDSS) is given by York et al. (2000). Details of the survey calibration are provided by Hogg et al. (2001), Smith et al. (2002), and Tucker et al. (2006). The data processing and quality assessment are discussed by Ivezić et al. (2004), and the photometric pipeline is described by Lupton et al. (1999).

The SDSS-II Supernova Survey was carried out during the Fall (September-November) of 2005-2007, using the $2.5 \mathrm{~m}$ telescope (Gunn et al. 2006) at Apache Point Observatory (Sacramento Peak, New Mexico). Observations were obtained in the SDSS ugriz filters (Fukugita et al. 1996) with a wide-field CCD camera (Gunn et al. 1998), operating in time-delay-andintegrate (TDI, or drift scan) mode. The region of the sky covered by the SDSS-II Supernova Survey (designated Stripe 82; see Stoughton et al. 2002) was bounded by $-60^{\circ}<\alpha_{\text {J2000 }}<60^{\circ}$ and $-1.258<\delta_{\mathrm{J} 2000}<1.258$. On average, any given part of this $\sim 300 \mathrm{deg}^{2}$ area was imaged once every four days during the survey operations. 
Difference images were produced in the SDSS gri filter bands by subtracting template images, constructed from previous survey observations of the region, using an implementation of the methods described by Alard \& Lupton (1998). The difference images were searched for positive fluctuations using the DoPHOT photometry and object detection package (Schechter et al. 1993); typical limits (10 $\sigma$ above background) for the SDSS-II Supernova Survey were $g \approx 21.8, r \approx 21.5$, and $i \approx 21.2$ mag. A combination of software cuts and human visual inspection was then used to identify promising SN candidates from the full set of transient detections. As a key component of prioritizing SN candidates for follow-up spectroscopic observations, the light curves of SN candidates were fit to models of Type Ia, Type Ib/c, and Type II SNe (see Filippenko 1997 for a review of SN types). This procedure is referred to as "photometric typing," and is described in detail by Sako et al. (2008).

Spectroscopic observations for both SN classification and redshift determination were provided by a number of different telescopes. The spectra of the SNe utilized in the present SN rate analysis were provided by the Hobby-Eberly $9.2 \mathrm{~m}$ at McDonald Observatory, the Astrophysical Research Consortium $3.5 \mathrm{~m}$ at Apache Point Observatory, the Hiltner $2.4 \mathrm{~m}$ at the MichiganDartmouth-MIT Observatory, the Subaru $8.2 \mathrm{~m}$ at the National Astronomical Observatory of Japan, the Keck-I $10 \mathrm{~m}$ at the W. M. Keck Observatory, the Mayall $3.8 \mathrm{~m}$ at Kitt Peak National Observatory, the $3.5 \mathrm{~m}$ ESO New Technology Telescope (NTT) at the European Southern Observatory, the SALT $11 \mathrm{~m}(9.5 \mathrm{~m}$ clear aperture) at the South African Astronomical Observatory, and the $2.6 \mathrm{~m}$ Nordic Optical Telescope, $3.5 \mathrm{~m}$ Telescopio Nazionale Galileo, and $4.2 \mathrm{~m}$ William Herschel Telescope at the Observatorio del Roque de los Muchachos. Details of the SDSSII Supernova Survey spectroscopic data reductions are given by Zheng et al. (2008). Comparison to high-quality SDSS galaxy spectra shows that $\mathrm{SN}$ spectroscopic redshifts are accurate to $\sim 0.0005$ when galaxy emission features are used and to $\sim 0.005$ when SN features are used. In either case, the uncertainties in the spectroscopic SN redshifts are negligible for the SN-rate studies considered here.

While the difference imaging pipeline used during the SN search provides initial photometric measurements, subsequent to the search more precise SN photometry is provided using a scene modeling photometry (SMP) technique developed by Holtzman et al. (2008). The final analysis of SN light curves and the selection cuts used to define the $\mathrm{SN}$ rate sample discussed in this paper are based on SMP.

\section{SN Ia SAMPLE FOR THE RATE MEASUREMENT}

\subsection{SN Selection Requirements}

For the SDSS-II Supernova Survey measurement of the low-redshift SN Ia rate (Dilday et al. 2008), we included in the SN Ia sample all spectroscopically confirmed SNe Ia at $z<0.12$, subject to a set of objective selection criteria that can be robustly modeled with our SN Monte Carlo (MC) simulations. To account for spectroscopic incompleteness, we used the MLCS2k2 SN Ia model (Jha et al. 2007) to analyze the SMP (Section 2) light curves for a set of $\sim 1000$ photometric SN candidates, which comprised $\sim 500$ "best" SN Ia candidates and $\sim 500$ randomly chosen $\mathrm{SN}$ candidates.

In the present analysis, we adopt a somewhat different approach to selecting the SN sample for use in measurement of the $\mathrm{SN}$ rate. Rather than focusing on the low-redshift $(z<0.12)$
SNe, which can be demonstrated to be a complete sample, we define objective selection criteria for $\mathrm{SNe}$ Ia at all redshifts, and determine the completeness of the resulting samples based on analysis of simulated samples of SNe. As discussed in Section 2, during the survey the search-photometry light curves of SN candidates were fit to models of Type Ia, Type Ib/c, and Type II SNe, and the results were used as a factor in prioritizing our spectroscopic follow-up resources.

In addition, as a method of searching for photometric $\mathrm{SNe}$ Ia subsequent to the survey, the search-photometry light curves were used to define a Bayesian probability for each SN candidate to be an $\mathrm{SN}$ of a given type. This was done by marginalizing over the light-curve fit parameters to obtain the Bayesian evidence and requiring that the evidence for the three SN types sums to 1. This defines the "probability," $p_{\mathrm{T}}$, for an object to be an SN of type T. This quantity can be considered a probability in the sense that it is bounded by $0<p_{\mathrm{T}}<1$, and is normalized to $1\left(\Sigma_{\mathrm{T}} p_{\mathrm{T}}=1\right)$. However, this procedure makes the initial assumption that the object is a SN (i.e., that the three types $\mathrm{T}=\mathrm{Ia}, \mathrm{Ib} / \mathrm{c}$, II are exhaustive), and does not allow for other possibilities for the identity of the object (e.g., active galactic nucleus). Despite this caveat, the quantities $p_{\mathrm{T}}$ are useful statistics for analyzing the search photometry light curves. The procedure is motivated by, and modeled after, that discussed by Kuznetsova \& Connolly (2007) and Poznanski et al. (2007a).

The selection criteria for SN candidates that we impose on the photometric-typing fits (Section 2) to the search photometry light curves are as follows.

1. Bayesian $p_{\text {Ia }}>0.45$.

2. At least three search-discovery epochs.

3. If the candidate has more than five search-photometry epochs, the best-fit SN Ia model is not SN 2005gj.

These selection criteria were determined by correlating the fit results from the full analysis of the SMP light curves for the $\sim 1000$ photometric SN candidates from the 2005 season with statistics of the corresponding photometric-typing fits to the search photometry, and looking for a combination of cuts that would result in a sample of SN candidates with high purity and completeness with respect to $\mathrm{SNe}$ Ia. Several possible statistics of the photometric-typing fits were considered to see whether they would improve the efficiency for selecting $\mathrm{SNe}$ Ia from the search-photometry SN candidates. The conclusion of these correlation studies was that the Bayesian probability, $p_{\text {Ia }}$, is the best single quantity to consider for selecting a large fraction of SNe Ia, and no significant improvement was found by considering additional fit statistics, such as the value of the reduced $\chi^{2}$ statistic for the fit.

The peculiar SN Ia 2005gj, which has a flat light curve after maximum brightness (Aldering et al. 2006; Prieto et al. 2007), is included as one of the SN Ia light-curve models in the photometric-typing fits. The requirement that the best-fit SN not be SN 2005gj is effectively intended to remove both peculiar $\mathrm{SNe}$ Ia and other non-SN transients, such as active galactic nuclei. Some core-collapse $\mathrm{SNe}$ are well fit by the broad light curve of SN 2005gj, and this cut also serves as a way for rejecting these from our SN Ia sample selection. Search-discovery epochs refer to epochs for which the transient object was detected by the survey difference imaging and object detection pipeline (Sako et al. 2008).

This sample selection, based on the photometric-typing procedure, resulted in $\sim 600 \mathrm{SN}$ Ia candidates per observing season of the SDSS-II Supernova Survey. SMP was then generated for 
Table 1

Number of SNe Ia for Rate Measurement

\begin{tabular}{lrrrr}
\hline \hline $\begin{array}{l}\text { Redshift } \\
\text { Limit }\end{array}$ & Confirmed & $\begin{array}{c}\text { Photometric } \\
\text { (Spect- } z)\end{array}$ & $\begin{array}{c}\text { Photometric } \\
\text { (Photo-z) }\end{array}$ & Total \\
\hline 0.15 & $72(91 \%)$ & $5(6 \%)$ & $2(3 \%)$ & 79 \\
0.20 & $140(74 \%)$ & $35(18 \%)$ & $15(8 \%)$ & 190 \\
0.25 & $217(62 \%)$ & $76(22 \%)$ & $57(16 \%)$ & 350 \\
0.30 & $270(52 \%)$ & $113(22 \%)$ & $133(26 \%)$ & 516 \\
$\infty$ & $312(40 \%)$ & $148(19 \%)$ & $314(41 \%)$ & 774
\end{tabular}

these candidates, producing more reliable photometry and providing measurements at additional observing epochs, compared with the SN search photometry. In addition to the requirements on the photometric-typing fits, we require the SMP light curves for the candidates to satisfy similar selection criteria on lightcurve sampling and fit quality to those discussed by Dilday et al. (2008). We list these criteria below.

1. $-51^{\circ}<\alpha_{\mathrm{J} 2000}<57^{\circ}$.

2. There are photometric observations on at least five separate epochs between -20 days and +60 days relative to $B$-band peak light in the $\mathrm{SN}$ rest frame.

3. At least one epoch with signal-to-noise ratio $>5$ in each of $g, r$, and $i$ (not necessarily the same epoch in each passband).

4. At least one photometric observation at least two days before $B$-band peak light in the $\mathrm{SN}$ rest frame.

5. At least one photometric observation at least 10 days after $B$-band peak light in the $\mathrm{SN}$ rest frame.

6. MLCS2k2 light-curve fit probability $>0.001$.

7. MLCS2k2 light-curve fit parameter $\Delta>-0.4$.

The first requirement states that the $\mathrm{SN}$ is within the rightascension range of the calibration-star catalog. The second and third requirements ensure that the object is a significant and authentic astrophysical transient. The fourth and fifth requirements are imposed so that we have a robust measurement of the time of maximum brightness for the $\mathrm{SN}$ candidates as well as a reliable measurement of the light-curve decline, which is useful for rejecting Type II SNe. The sixth requirement is used to reject peculiar SNe Ia that are not well represented by the MLCS2k2 light-curve model, as well as non-SN Ia transient objects. The seventh requirement is additionally used to reject objects with flat light curves such as $\mathrm{SNe}$ II and active galactic nuclei. The low-redshift SN data used to define the MLCS2k2 model only exhibit values of the light-curve shape parameter $\Delta \gtrsim-0.35$, so a cut at $\Delta>-0.4$ specifies that the object is within the valid range of the MLCS2k2 model, with some allowance for measurement error.

\subsection{SN Sample}

Over the entire redshift range of $\mathrm{SNe}$ discovered by the SDSS-II Supernova Survey $(z \lesssim 0.45)$, there are 774 SN Ia candidates (312 spectroscopically confirmed) that satisfy the selection criteria above. The redshift distribution for these SNe is shown in Figure 1. However, as will be discussed in Section 4, the systematic uncertainty related to our sample selection becomes dominant for $z \gtrsim 0.2$, thereby reducing our ability for making precise SN Ia rate measurements. The numbers of $\mathrm{SNe}$ for several values of the maximum redshift are given in Table 1. Spectroscopically confirmed SNe Ia from this sample are listed in Table 2.

$\mathrm{SNe}$ for which we have photometric observations of the light curve, but do not have any spectroscopic observations to determine the spectral type of the $\mathrm{SN}$, are referred to as

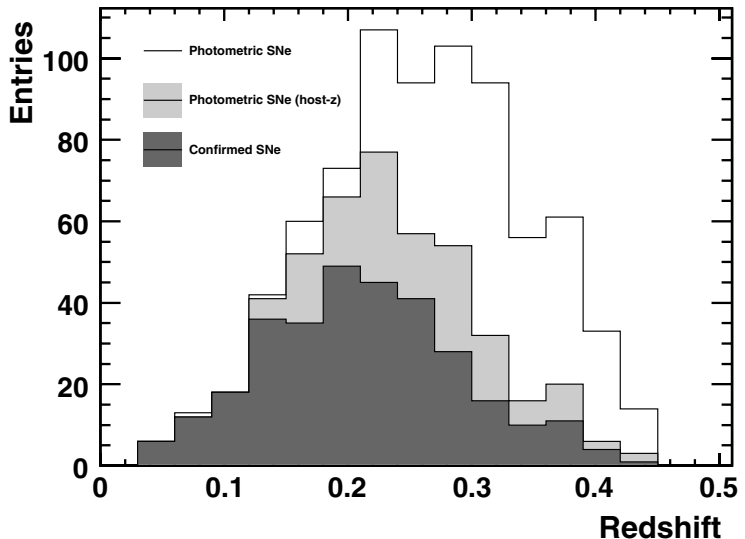

Figure 1. Redshift distribution for the 774 SDSS-II Supernova Survey SNe passing all selection criteria. The dark gray, light gray, and white shading represent spectroscopically confirmed $\mathrm{SNe}$ Ia, photometric SNe with measured host-galaxy redshifts, and photometric SNe without measured host-galaxy redshifts, respectively.

Table 2

Confirmed SNe Ia in the Rate Sample

\begin{tabular}{rcrccc}
\hline \hline SN ID & IAU Name & $\begin{array}{c}\alpha_{\text {J2000 }} \\
(\mathrm{deg})\end{array}$ & $\begin{array}{c}\delta_{\text {J2000 }} \\
(\mathrm{deg})\end{array}$ & Redshift & Fitprob \\
\hline 762 & $2005 \mathrm{eg}$ & 15.53518 & -0.87907 & 0.191 & 0.945 \\
1032 & $2005 \mathrm{ez}$ & 46.79565 & +1.11952 & 0.130 & 0.541 \\
1112 & $2005 \mathrm{fg}$ & 339.01761 & -0.37527 & 0.258 & 0.792 \\
1166 & $\ldots$ & 9.35560 & +0.97320 & 0.382 & 0.992 \\
1241 & $2005 \mathrm{ff}$ & 337.67249 & -0.77664 & 0.090 & 0.988 \\
1253 & $2005 \mathrm{fd}$ & 323.79895 & +0.16305 & 0.262 & 0.834 \\
1371 & $2005 \mathrm{fh}$ & 349.37375 & +0.42929 & 0.119 & 0.995 \\
1580 & $2005 \mathrm{fb}$ & 45.32296 & -0.64412 & 0.183 & 1.000 \\
1688 & $\ldots$ & 321.35767 & +0.32447 & 0.359 & 0.183 \\
2017 & $2005 \mathrm{fo}$ & 328.94327 & +0.59343 & 0.262 & 0.972 \\
2031 & $2005 \mathrm{fm}$ & 312.04312 & -1.17149 & 0.153 & 0.894 \\
2165 & $2005 \mathrm{fr}$ & 17.09165 & -0.09639 & 0.288 & 0.930 \\
2246 & $2005 \mathrm{fy}$ & 50.09031 & -0.88564 & 0.195 & 0.904 \\
2308 & $2005 \mathrm{ey}$ & 34.27273 & +0.28020 & 0.148 & 0.960 \\
\hline
\end{tabular}

(This table is available in its entirety in a machine-readable form in the online journal. A portion is shown here for guidance regarding its form and content.)

photometric SNe. Photometric SNe fall into two classes: (1) those that have a precisely (i.e., spectroscopically) measured redshift for their host galaxy and (2) those that do not have a precisely measured redshift for their host galaxy. When the redshift for a photometric SN candidate is unknown, the candidate light curve is analyzed with the "photo- $z$ " option in the flux-based MLCS2k2 light-curve fitter (Dilday et al. 2008). To fit SN light curves for redshift, we assume a cosmological model, and hence a distance versus redshift relation, in order to take advantage of knowledge of the absolute magnitude of SNe Ia. In addition to redshift, the SN light curves are fit for the time of maximum (in the SN rest-frame $B$ band), the luminosity parameter $\Delta$, and the extinction parameter $A_{V}$; see Jha et al. (2007) for a comprehensive discussion of MLCS2k2.

To investigate the accuracy and precision of the photo- $z$ fits, we carry out photo- $z$ fits to the spectroscopically confirmed $\mathrm{SNe}$ Ia, as well as to the photometric SNe Ia with spectroscopically measured redshifts of $z<0.15$. At such low redshifts, this is essentially a complete sample of SNe Ia. A plot of the residuals for the photometric redshifts is shown in Figure 2, illustrating that the SN photo- $z$ fits are negligibly biased, and accurate to $\sim 0.01$ at low redshift. The numbers of SNe from categories (1) and (2) that satisfy the selection criteria are given, for several 


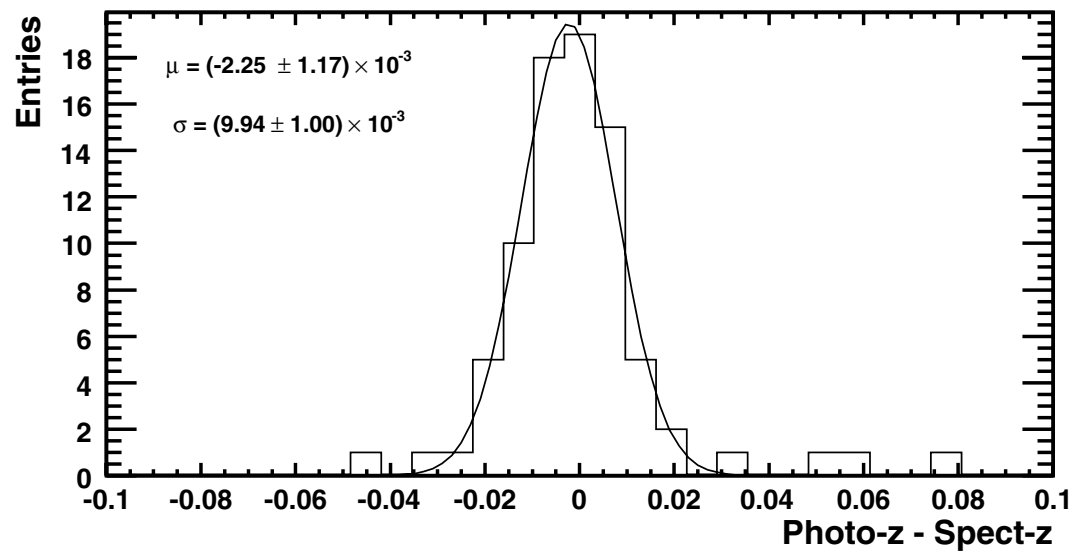

Figure 2. Redshift residuals (photo-z minus spectroscopic-z) for photo- $z$ fits to a sample of spectroscopically confirmed SNe Ia and photometric SNe Ia with spectroscopic redshifts at $z<0.15$. The best-fit Gaussian function is overlaid. Here, $\mu$ refers to the mean and $\sigma$ to the standard deviation of the best-fit function. This plot illustrates that the SN photo- $z$ fits are negligibly biased and accurate to $\sim 0.01$ at low redshift.

Table 3

SNe with Spectroscopic Redshifts in the Rate Sample

\begin{tabular}{rrccc}
\hline \hline SN ID & $\begin{array}{c}\alpha_{\text {J2000 }} \\
(\mathrm{deg})\end{array}$ & $\begin{array}{c}\delta_{\text {J2000 }} \\
(\mathrm{deg})\end{array}$ & Redshift & Fitprob \\
\hline 703 & 336.21786 & +0.65059 & 0.300 & 0.976 \\
779 & 26.67369 & -1.02072 & 0.238 & 0.986 \\
911 & 38.69067 & -0.11571 & 0.208 & 0.930 \\
1008 & 28.27810 & +1.11369 & 0.226 & 0.974 \\
1415 & 6.10647 & +0.59921 & 0.212 & 0.924 \\
1740 & 5.40428 & -0.88099 & 0.167 & 0.013 \\
2057 & 320.39969 & -0.31708 & 0.212 & 0.512 \\
2081 & 337.30505 & -1.20783 & 0.252 & 0.753 \\
2162 & 15.44242 & -0.13368 & 0.173 & 0.777 \\
2532 & 27.74742 & -0.23427 & 0.270 & 0.913 \\
2632 & 45.59013 & -1.22610 & 0.296 & 0.044 \\
2639 & 330.46411 & +0.66447 & 0.215 & 0.278 \\
2734 & 48.20686 & -0.69485 & 0.303 & 0.149 \\
2806 & 45.26690 & +0.27364 & 0.301 & 0.340 \\
\hline
\end{tabular}

(This table is available in its entirety in a machine-readable form in the online journal. A portion is shown here for guidance regarding its form and content.)

values of limiting redshift, in Table 1. Photometric SNe from categories (1) and (2) are listed in Tables 3 and 4, respectively.

\subsection{Bias Correction for the Observed Redshift Distribution}

The observed redshift distributions for the SNe Ia from the SDSS-II Supernova Survey that satisfy the selection criteria of Section 3.1 are shown in Figure 1. As the number distribution is not constant with redshift, nonzero measurement error of the redshifts will result in a bias in the measured number distribution. In general, the observed number distribution as a function of redshift, $\eta(z)$, is related to the true redshift distribution, $\eta_{0}(z)$, through

$$
\eta(z)=\int_{-\infty}^{\infty} \eta_{0}\left(z^{\prime}\right) p\left(z \mid z^{\prime}\right) d z^{\prime},
$$

where $p\left(z \mid z^{\prime}\right)$ is the probability that an $\mathrm{SN}$ at redshift $z^{\prime}$ will have a measured redshift of $z$. The number distribution, $\eta_{0}(z)$, is related to the volumetric rate, $r_{V}(z)$, and the redshift dependent efficiency, $\epsilon(z)$, through

$$
\eta_{0}(z) d z \propto \frac{r_{V}(z) \epsilon(z)}{1+z} \frac{d V}{d z} d z
$$

where $d V / d z$ is the volume element at redshift $z$. In what follows, we use a Gaussian approximation for $p\left(z \mid z^{\prime}\right)$, which
Table 4

SNe with Photometric Redshifts in the Rate Sample

\begin{tabular}{rrcccc}
\hline \hline SN ID & $\begin{array}{c}\alpha_{\text {J2000 }} \\
(\mathrm{deg})\end{array}$ & $\begin{array}{c}\delta_{\text {J2000 }} \\
(\mathrm{deg})\end{array}$ & Redshift & Redshift Error & Fitprob \\
\hline 822 & 40.56070 & -0.86217 & 0.220 & 0.018 & 0.090 \\
1342 & 346.52740 & +0.11688 & 0.283 & 0.026 & 0.962 \\
1403 & 359.70386 & +0.43188 & 0.341 & 0.034 & 0.112 \\
1658 & 357.50443 & +0.65006 & 0.256 & 0.025 & 0.836 \\
1899 & 323.34305 & -0.70642 & 0.341 & 0.037 & 0.005 \\
2784 & 28.07526 & -0.04169 & 0.381 & 0.021 & 0.488 \\
2855 & 16.17518 & -0.35642 & 0.233 & 0.016 & 0.978 \\
3206 & 13.57741 & +0.41816 & 0.387 & 0.031 & 0.003 \\
3368 & 44.45594 & +1.23082 & 0.320 & 0.035 & 0.831 \\
3417 & 314.27606 & +0.97825 & 0.262 & 0.016 & 0.413 \\
3506 & 336.25064 & -0.97821 & 0.211 & 0.011 & 0.963 \\
3945 & 346.00906 & -0.28307 & 0.260 & 0.017 & 0.070 \\
3975 & 29.82097 & +0.20364 & 0.399 & 0.019 & 0.002 \\
3983 & 7.27582 & -0.25663 & 0.279 & 0.021 & 0.220 \\
\hline
\end{tabular}

(This table is available in its entirety in a machine-readable form in the online journal. A portion is shown here for guidance regarding its form and content.)

in most cases is a good representation of the SDSS-SN photo- $z$ errors,

$$
p\left(z \mid z^{\prime}\right)=\frac{1}{\sqrt{2 \pi} \sigma\left(z^{\prime}\right)} e^{-\left(z-z^{\prime}\right)^{2} / 2 \sigma^{2}\left(z^{\prime}\right)},
$$

where $\sigma\left(z^{\prime}\right)$ is the standard deviation of the SN photo- $z$. To determine $\sigma\left(z^{\prime}\right)$, we perform photo- $z$ fits for all SNe that pass our selection criteria and then fit a power law for the typical error in the photo- $z$. The photo- $z$ error as a function of fitted photo- $z$ is shown in Figure 3, along with the best-fit power law, $\sigma(z)=A z^{k}$. The best fit has $A \approx 0.2$ and $k \approx 1.5$.

To estimate the bias in the observed SN number distribution, we integrate both $\eta(z)$ and $\eta_{0}(z)$ for a range of SN rate models, $r(z) \propto(1+z)^{v}$, over the redshift bins shown in Figure 1. The resulting bias, defined as $\Delta N / N=\left(N-N_{0}\right) / N$, where $N$ is the number of observed $\mathrm{SNe}$ and $N_{0}$ is the number of underlying $\mathrm{SNe}$ in each bin, is at the few percent level and is shown in Figure 4. We note that we have considered here a hypothetical SN sample where all the redshifts are determined photometrically. The bias in the observed SDSSII Supernova Survey SN distribution will be much smaller, since many of the redshifts are determined spectroscopically. In Table 5 we list the bias correction appropriate for our bestfit power-law SN rate model, $r_{V}(z) \propto(1+z)^{2.04}$, computed over bins in redshift of width $\delta z=0.05$. The bias due to the 


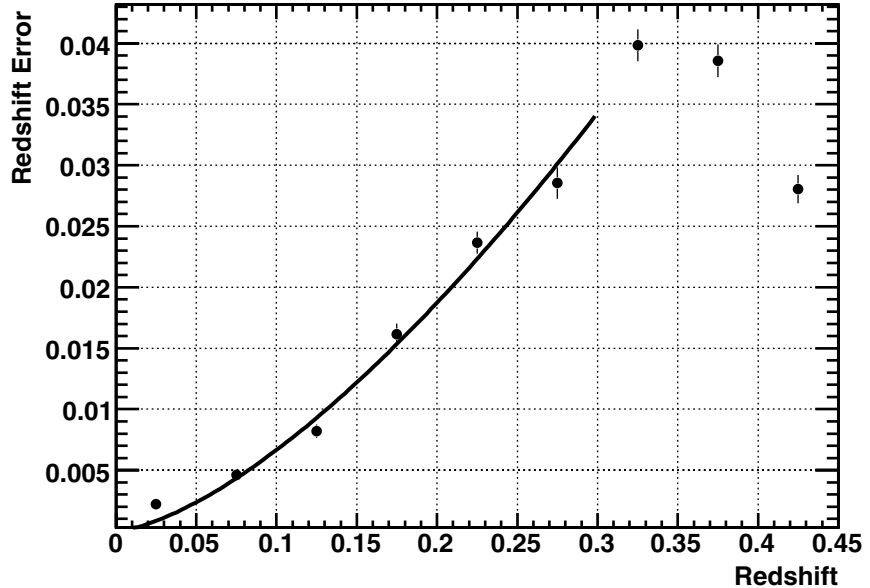

Figure 3. Standard deviation of the SN photo- $z$ estimates as a function of fitted photo- $z$. The points show the mean value of the photo- $z$ error, in bins of width $\delta z=0.05$. The error bars represent the uncertainty in the mean, and the solid line represents the best-fit power law for the interval $0<z<0.3$.

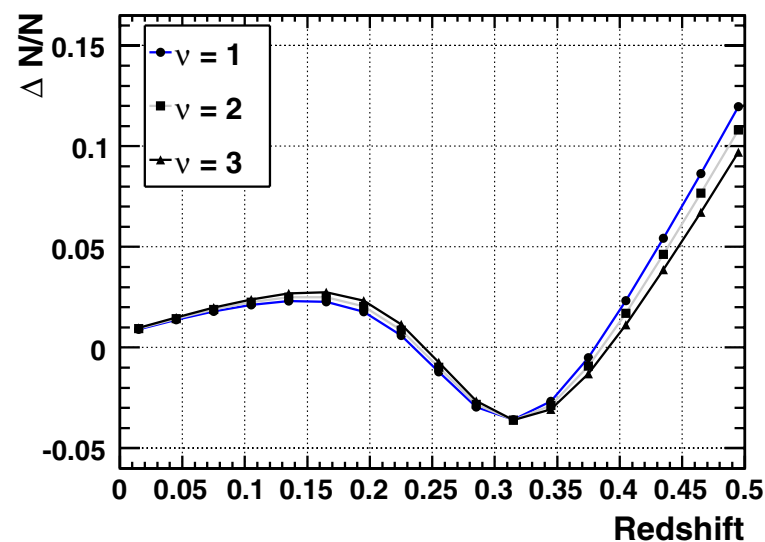

Figure 4. Bias, $\Delta N / N$, in an observed distribution of SNe, with a typical error in the measured redshifts (Section 3.3) and discovery efficiency (Section 3.1) as determined for the SDSS-II Supernova Survey. The bias is plotted for a range of power-law SN rate models, $r(z)=A_{p}(1+z)^{v}$.

(A color version of this figure is available in the online journal.)

use of SN photometric redshifts is negligible in comparison to the statistical and systematic uncertainties on the SN Ia rate measurements. Additionally, we note that in Section 5.2 we fit the SDSS-II Supernova Survey data to models of the SN rate using an unbinned maximum likelihood that properly accounts for the bias discussed here.

\subsection{Estimating Contamination from Non-Ia SNe}

In studies of SNe Ia that include photometrically identified $\mathrm{SNe}$, one must correct for contamination from non-Ia (mainly core-collapse) $\mathrm{SNe}$ and evaluate the corresponding systematic uncertainty. Non-Ia SNe form a less homogeneous set than Type Ia $\mathrm{SNe}$ and, in contrast to $\mathrm{SNe} \mathrm{Ia}$, no general parametric models exist to describe the light curves of non-Ia SNe. In addition, owing to the strong interest in the use of SNe Ia as cosmological distance indicators, there is often an explicit observational bias against spectroscopic follow-up observations of non-Ia SNe in modern SN surveys (e.g., Sullivan et al. 2006a; Sako et al. 2008). As the global set of well-observed $\mathrm{SNe}$ Ia has grown, this limitation for future SN Ia studies has been recognized, and non-Ia SN observations, in part to better characterize the underlying $\mathrm{SN}$ population, are gaining increased attention (e.g., Gal-Yam et al. 2005, 2007).

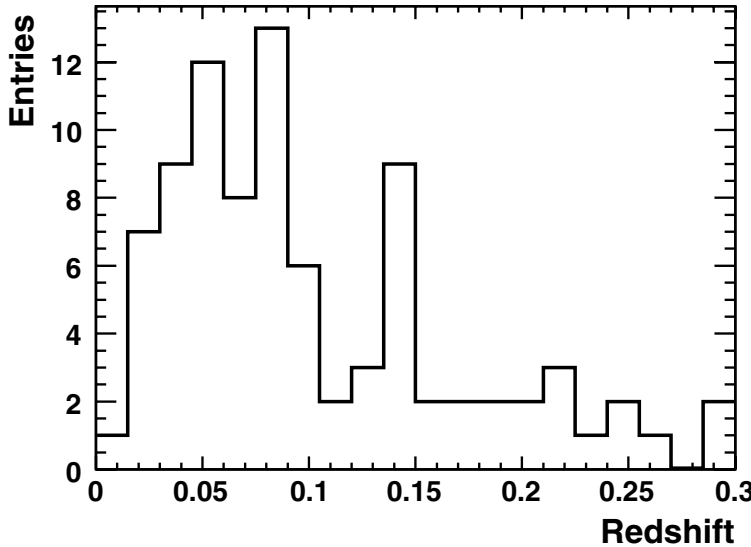

Figure 5. Redshift distribution for spectroscopically confirmed non-Ia SNe for the 2005-2007 observing seasons of the SDSS-II Supernova Survey.

Table 5

SN Rate versus Redshift

\begin{tabular}{cccc}
\hline \hline Redshift & $\begin{array}{c}\mathrm{SN} \mathrm{Rate}^{\mathrm{a}} \\
\left(10^{-5} \mathrm{SNe} \mathrm{yr}^{-1} \mathrm{Mpc}^{-3} h_{70}^{3}\right)\end{array}$ & $-\Delta N / N^{\mathrm{b}}$ & $N_{\mathrm{CC}} / N_{\text {Ia }}$ \\
\hline $0.025-0.050$ & $2.78_{-0.83-0.00}^{+1.12+0.15}$ & $0.00 \%$ & $\ldots$ \\
$0.075-0.125$ & $2.59_{-0.44-0.01}^{+0.52+0.18}$ & $-0.06 \%$ & $0.71_{-0.33}^{+0.56} \%$ \\
$0.125-0.175$ & $3.07_{-0.34-0.05}^{+0.38+0.35}$ & $-0.21 \%$ & $2.99_{-1.40}^{+2.37} \%$ \\
$0.175-0.225$ & $3.48_{-0.30-0.07}^{+0.32+0.82}$ & $-0.21 \%$ & $2.71_{-1.27}^{+2.14} \%$ \\
$0.225-0.275$ & $3.65_{-0.28-0.12}^{+0.31+1.82}$ & $+0.28 \%$ & $2.06_{-0.96}^{+1.63} \%$ \\
$0.275-0.325$ & $4.34_{-0.34-0.16}^{+0.37+3.96}$ & $+1.86 \%$ & $0.67_{-0.31}^{+0.53} \%$ \\
\hline
\end{tabular}

Notes.

a The errors given are statistical and systematic, respectively.

b Assuming a rate model $r_{V} \propto(1+z)^{2.04}$; see Section 3.3.

For these reasons, it is difficult to quantify contamination of the set of photometric SN Ia candidates from non-Ia $\mathrm{SNe}$ in the same way that we have treated determination of the completeness of the SN Ia sample-namely, by modeling the SN survey observations of the underlying population with our SN MC simulations. To place limits on the expected level of contamination of the photometric SN sample by non-Ia SNe, we instead consider the set of spectroscopically confirmed nonIa SNe from the SDSS-II Supernova Survey. There are 42 spectroscopically confirmed non-Ia SNe from the first two years of the SDSS-II Supernova Survey, and an additional 45 from the third year. The reason for the greater relative number of non-Ia $\mathrm{SNe}$ in the third year is that, in addition to the usual SN Ia search, the third season included spectroscopic observing time on the Subaru telescope specifically allocated for Type II SNe (D'Andrea et al. 2010). The redshift distribution for the spectroscopically confirmed non-Ia SNe from all three years of the SDSS-II Supernova Survey is shown in Figure 5.

To investigate possible contamination from non-Ia SNe, we apply to the set of non-Ia SNe the same light-curve fits (to an SN Ia model) and selection criteria that are used in defining the SN Ia sample. As the set of photometric SN Ia candidates includes SNe both with and without spectroscopically measured redshifts, we consider contamination from both distance and photo- $z$ fits for the confirmed non-Ia SN sample. If we fix the redshift to its spectroscopically determined value and fit the set of non-Ia SNe for distance, two events satisfy the selection criteria for the SN Ia rate sample. These are SDSS-SN 14492 (SN 2006jo), an SN Ib at $z=0.077$, and SDSS-SN 17422 (no IAU designation), a SN II at $z=0.149$. The light-curve fits using the MLCS2k2 SN Ia model for these SNe are shown in Figure 6. 

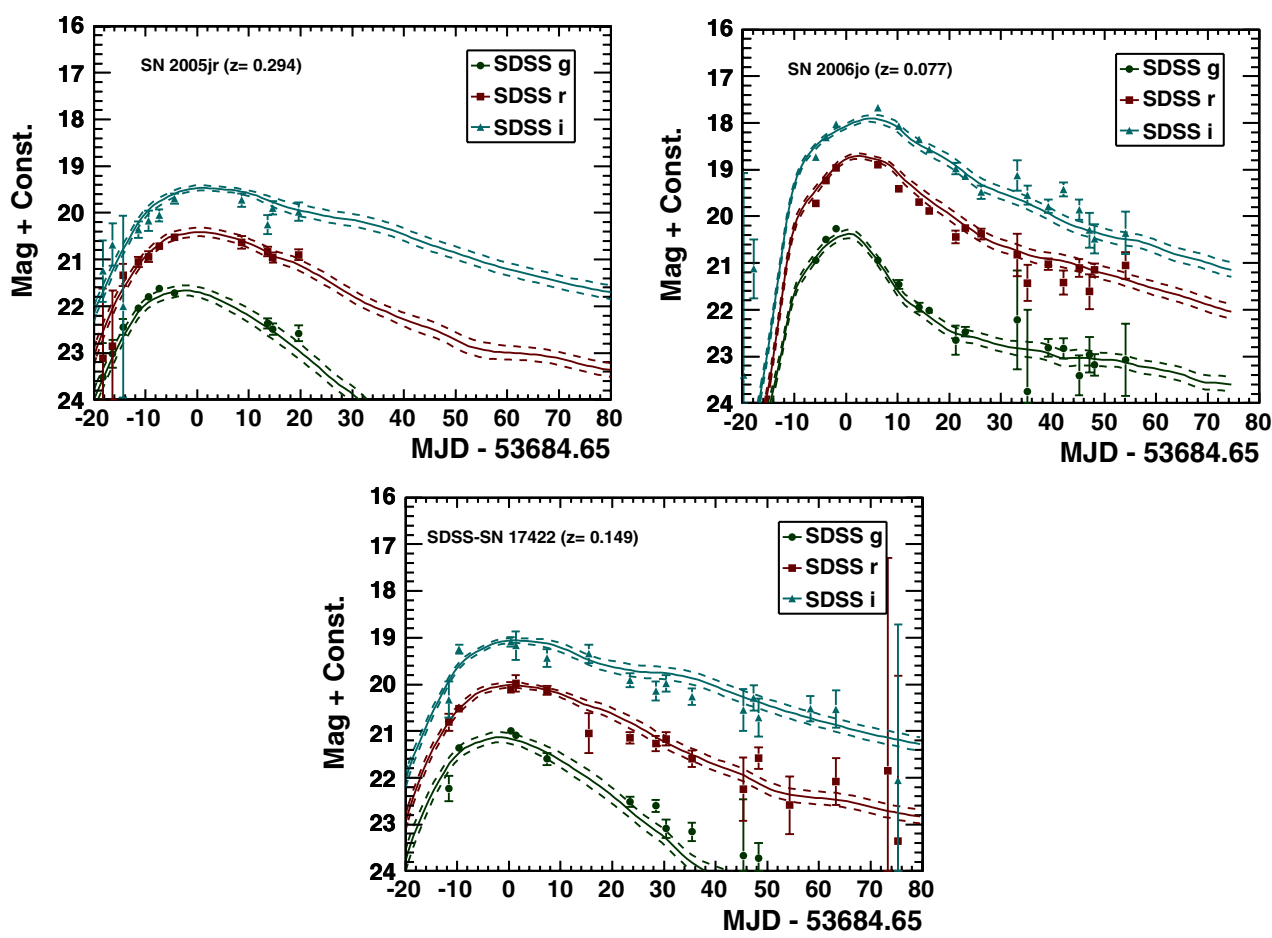

Figure 6. Photo- $z$ light-curve fits, to an SN Ia model, for the non-Ia SNe SDSS-SN 8679 (top left panel), SDSS-SN 14492 (top right panel), and SDSS-SN 17422 (bottom right panel). The points represent the observed SN magnitudes, as a function of time, in the observer frame. The solid lines represent the best-fitting model light curves, for the SDSS $g, r$, and $i$ filter bands, and the dashed lines represent the corresponding $1 \sigma$ MLCS2k2 model errors. For clarity, the $g, r$, and $i$ light curves are offset by $+0,+1$, and +2 mag, respectively.

(A color version of this figure is available in the online journal.)

If we instead fit the set of non-Ia SNe for photo-z, three of them satisfy the selection criteria for the SN Ia rate sample. These include the two non-Ia SNe mentioned above, as well as SDSSSN 8679 (SN 2005jr), an SN IIn at $z=0.294$. If we assume that the fraction of non-Ia SNe that satisfy the selection criteria, $q_{\mathrm{CC}}$, is a random variable that follows a binomial distribution, then an observation of two successful events out of 87 in total gives $1 \sigma$ limits on $q_{\mathrm{CC}}$ of $0.023_{-0.015}^{+0.030}$. An observation of three successful events gives $1 \sigma$ limits on $q_{\mathrm{CC}}$ of $0.035_{-0.019}^{+0.032}$.

Some care must be taken when interpreting the fits to the spectroscopically confirmed non-Ia $\mathrm{SNe}$ as an estimate of the false-positive rate. The spectroscopic incompleteness of the SDSS-II Supernova Survey for non-Ia SNe is not well constrained, and the set of spectroscopically confirmed nonIa $\mathrm{SNe}$ is manifestly not complete. As mentioned above, the distribution of non-Ia SN light-curve properties is not well measured, so it is difficult to evaluate whether these non-Ia $\mathrm{SNe}$ are a representative sampling of the underlying population. However, as the SDSS-II Supernova Survey has a built-in selection bias against non-Ia SNe (Sako et al. 2008), it can plausibly be claimed that any bias in the SDSS-II Supernova Survey non-Ia sample is a bias toward the most "SN Ia-like" non-Ia SNe. With that being the case, our estimate of $\sim 3 \%$ probability for non-Ia SNe to satisfy our selection criteria can be considered a conservative upper bound.

To turn this into an estimate of the contamination of the photometric SN sample from non-Ia SNe, we must also make an estimate of the non-Ia SN rate. In the redshift range containing the majority of photometric SNe from the SDSS-II Supernova Survey, $0.2<z<0.3$, the ratio of the non-Ia SN rate to the SN Ia rate has been measured by Botticella et al. (2008) as $\left(r_{\mathrm{CC}} / r_{\mathrm{Ia}}\right)_{z=0.25}=5.6 \pm 3.5$ and by Bazin et al. (2009) as $\left(r_{\mathrm{CC}} / r_{\mathrm{Ia}}\right)_{z=0.30}=4.5 \pm 1.0$. Assuming the ratio is constant for $0.2<z<0.3$ and combining the two measurements gives a ratio of the non-Ia $\mathrm{SN}$ rate to the $\mathrm{SN}$ Ia rate of $r_{\mathrm{CC}} / r_{\mathrm{Ia}}=4.6 \pm 1.0$.

Furthermore, the above estimate of $\sim 3 \%$ of non-Ia $\mathrm{SNe}$ satisfying the SN Ia selection criteria was determined for $\mathrm{SNe}$ that were detected by the SDSS-II Supernova Survey and some estimate must be made of the detection efficiency. To estimate the ratio of detection efficiency for non-Ia $\mathrm{SNe}$ versus SNe Ia, we employ the following procedure. The efficiency for $\mathrm{SNe}$ Ia to satisfy the MLCS2k2 component of the selection function (i.e., the items listed in Section 3.1) as a function of redshift, shown in Figure 8, is transformed to an efficiency as a function of observer-frame peak magnitude by assuming a typical peak absolute magnitude for SNe Ia of $M_{B}=-19.3$, and a distance modulus derived from a standard $\Lambda \mathrm{CDM}$ cosmological model. This is a good operational definition for detection, since the primary requirements of the MLCS2k2 selection criteria are requirements on the SN sampling and signal-to-noise ratios. Although non-Ia SNe clearly differ from SNe Ia in properties such as light-curve shapes and K-corrections, we will assume that the detection efficiency for non-Ia SNe can be described by the same function of observer-frame magnitude; the efficiency function can then be mapped back to an efficiency as a function of redshift, given an assumed absolute magnitude.

Richardson et al. (2002, 2006) give estimates of the typical peak absolute magnitude for $\mathrm{SNe} \mathrm{Ib} / \mathrm{c}$ as $M_{B}=-18.07$, for SNe II-P as $M_{B}=-16.98$, and for SNe II-L as $M_{B}=-18.17$. Assuming an absolute magnitude of $M_{B} \approx-18.0$ for non-Ia $\mathrm{SNe}$, the ratio of detection efficiencies for non-Ia $\mathrm{SNe}$ versus $\mathrm{SNe}$ Ia as a function of redshift is then computed, and is well approximated by a function $\epsilon_{\mathrm{CC}}^{D} / \epsilon_{\mathrm{Ia}}^{D}=1 /\left(1+e^{\left(z-z_{0}\right) / s_{z}}\right)$. The best-fit parameters are found to be $z_{0}=(0.204,0.213,0.211)$ and $s_{z}=(0.032,0.032,0.031)$ for the 2005, 2006, and 2007 


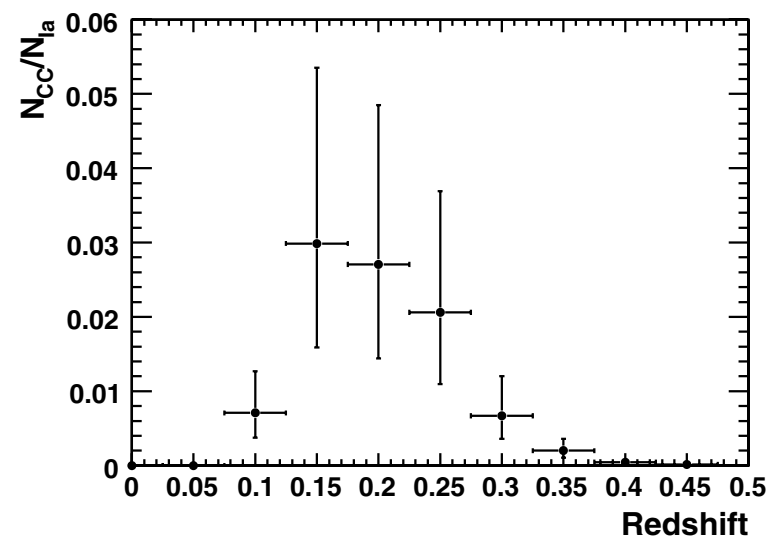

Figure 7. Estimated core-collapse contamination fraction vs. redshift.

seasons, respectively. The ratio of non-Ia to Ia SNe in the set of photometric $\mathrm{SNe}$ can then be estimated as

$$
\frac{N_{\mathrm{CC}}}{N_{\mathrm{Ia}}}=\frac{r_{\mathrm{CC}}}{r_{\mathrm{Ia}}} \frac{\epsilon_{\mathrm{CC}}^{q}}{\epsilon_{\mathrm{Ia}}^{q}} \frac{\epsilon_{\mathrm{CC}}^{D}}{\epsilon_{\mathrm{Ia}}^{D}},
$$

where $r$ is the $\mathrm{SN}$ rate, $\epsilon^{q}$ is the efficiency for an SN to satisfy the selection criteria on light-curve shape and fit probability, and $\epsilon^{D}$ is the efficiency for detection, as described above. We note that the ratio of core-collapse $\mathrm{SNe}$ to $\mathrm{SNe}$ Ia given by Equation (5) is a function of redshift. For the redshift range of interest, we take $r_{\mathrm{CC}} / r_{\mathrm{Ia}}=4.6 \pm 1.0$ as discussed above. The quantity $\epsilon_{\mathrm{CC}}^{q} / \epsilon_{\mathrm{Ia}}^{q}$ is $0.023_{-0.015}^{+0.030}$ for $\mathrm{SNe}$ with spectroscopically measured host-galaxy redshifts and $0.035_{-0.019}^{+0.032}$ for $\mathrm{SNe}$ without spectroscopically measured host-galaxy redshifts, as estimated from the spectroscopically confirmed non-Ia SNe in the SDSS-II Supernova Survey.

The estimated core-collapse contamination fraction as a function of redshift is shown in Figure 7, and the corresponding values are given in Table 5. The total estimated contamination of the SN sample by non-Ia SNe to a redshift limit of 0.3 is $2.04_{-0.95}^{+1.61} \%$. In the unbinned maximum likelihood fits discussed in Section 5.2, each photometric $\mathrm{SN}$ is given a weight according to the value of Equation (5).

\section{SURVEY EFFICIENCY}

The use of artificial SNe (fakes) in the survey discovery images and the SDSS-SN MC simulation to determine the SN discovery and selection efficiency have been discussed in detail by Dilday et al. (2008). For the MC simulation, for all observing epochs of the SDSS-II Supernova Survey, SN Ia photometry is generated based on an SN Ia light-curve model (MLCS2k2 in the present analysis), and the observing conditions corresponding to each epoch are used to generate realistic photometry errors. Characteristics of the simulated SN sample, such as distributions of time of maximum light, dust extinction, and intrinsic luminosity or decline rate, can be specified in order to simulate a realistic SN sample and to investigate systematic effects of variations in the underlying distributions.

Here we discuss the effect on the SN discovery efficiency of the modified selection procedure that uses statistics of the photometric-typing fits. To study the SN discovery efficiency for this SN rate analysis, we used the SDSS-SN MC simulation to generate a sample of $\sim 17,000 \mathrm{MC}$ SNe Ia, which comprises $\sim 1000 \mathrm{SNe}$ in each of 17 narrow redshift bins in the range $0.025<z<0.4$. These MC SNe were filtered through a simulation of the search detection efficiency. That is, the efficiencies as a function of signal-to-noise ratio determined from the fakes (Dilday et al. 2008) were applied to the simulated MC photometry. As in the search pipeline, a detection in at least two of the gri filters is required for the point to be considered to have been detected and to be included in the fit.

The simulated search photometry was then fit with the same photometric-typing code used during the search, and the cuts outlined in Section 3 were applied. The resulting selection efficiencies are shown in Figure 8.

As discussed by Sako et al. (2008), the photometric typing can be done with or without utilizing forced photometry (performing difference-imaging photometry at known positions of transient objects) and with or without a prior on the SN redshift (from the host-galaxy photometric or spectroscopic redshift measurement). The selection cuts are applied to fits that do not use forced photometry and do not use a prior on the SN redshift. While it is evident from examining the photometric-typing fits during the SN search campaign that utilizing forced photometry and/or a prior on the SN redshift in many cases improves the ability to distinguish SNe Ia based on their search-photometry light curves, it is significantly more difficult to model the selection function. The additional complications arise because forced photometry was applied nonuniformly to the SDSS-SN candidates, and because modeling the distribution of host-galaxy photometric redshifts and their errors is nontrivial (Oyaizu et al. 2008).

\subsection{Systematic Studies of the SN Discovery Efficiency}

In Dilday et al. (2008), we considered the effect on the SN rate discovery efficiency of variation in the distribution of SN population parameters and found that varying the distribution of extinction values had by far the largest effect. Here we repeat the systematic variation of the assumed extinction distribution with the modified selection procedure used in this paper. We vary the input extinction distribution, $p\left(A_{V}\right) \propto e^{-A_{V} / \tau}$, with $\tau=0.35 \pm 0.1$. The mean value and variation of $A_{V}$ are based upon investigation of the underlying $A_{V}$ distributions presented in the SDSS-SN cosmology analysis (Kessler et al. 2009). We find that for a low-extinction set of $\mathrm{SNe}(\tau=0.25)$, the efficiency differs negligibly from the default value of $\tau=0.35$. However, if the characteristic extinction is large $(\tau=0.45)$, the efficiency differs markedly from the fiducial set of SNe, particularly for $z \gtrsim 0.2$. Comparison of the efficiency between the fiducial and high-extinction assumptions is shown in Figure 8 (right panel). In Table 5, it can seen that the systematic uncertainty in the SN rate due to uncertainty in the extinction distribution becomes comparable to the statistical uncertainty for $z \approx 0.15$.

\section{SN Ia RATE RESULTS}

\subsection{Constant SN Ia Rate Model}

We first consider interpretations of the SN observations described above using a model of the SN rate that is constant as a function of redshift. In a constant-rate model, the volumetric rate is given by

$$
r_{V}=\frac{N}{\widehat{V T \epsilon}}
$$

where

$$
\widetilde{V T \epsilon}=\left(\Theta T_{\oplus}\right) \int_{z_{\min }}^{z_{\max }} \epsilon(z) \frac{d(V T / \Theta)}{d z} d z
$$



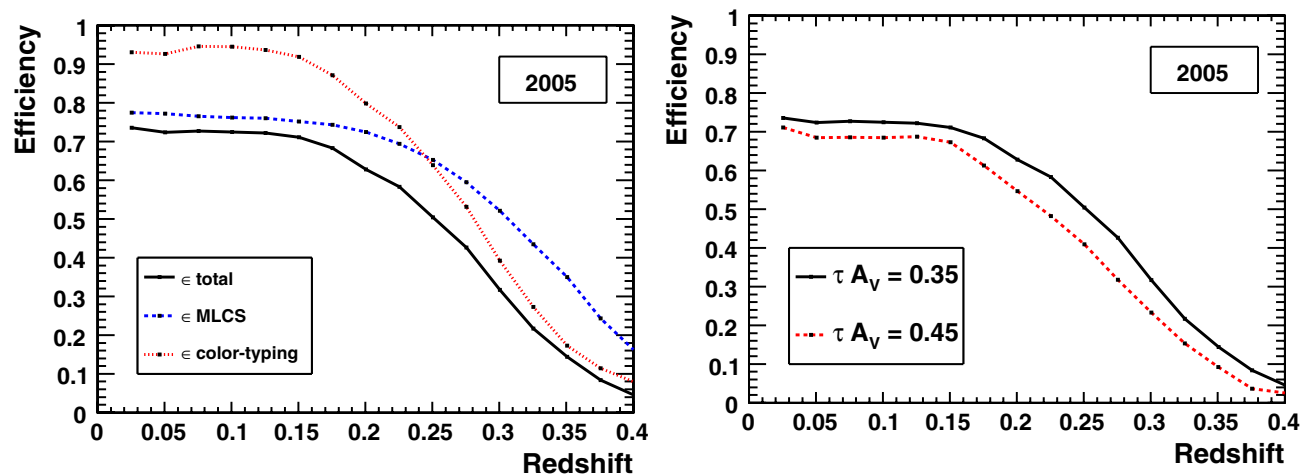

Figure 8. Results of studies of the SDSS-SN discovery efficiency, as a function of redshift, based on MC SN samples, for the 2005 observing season. The left panel shows the SN selection efficiency for the photometric-typing (red/dashed), MLCS2k2 (blue/dashed), and combined (black/solid) selection criteria. The right panel shows the efficiency for two different assumptions about the distribution of the extinction parameter, $A_{V}$. The $A_{V}$ distribution is assumed to have the form $e^{-A_{V} / \tau}$. The efficiency is shown for $\tau=0.35$ (default) and for $\tau=0.45$ ( $1 \sigma$ upper limit). The values for $\tau=0.25$ ( $1 \sigma$ lower limit) are not shown since they are indistinguishable from the $\tau=0.35$ values. The efficiency curves for the 2006 and 2007 observing seasons show similar behavior.

(A color version of this figure is available in the online journal.)

$N$ is the number of $\mathrm{SNe}$ in the sample, $T_{\oplus}$ is the observation time in the observer frame, $\Theta$ is the survey solid angle, $\epsilon(z)$ is the SN discovery efficiency, and $d(V T / \Theta) / d z$ is the element of volume multiplied by time per steradian in the $\mathrm{SN}$ frame. In the Friedmann-Robertson-Walker metric, $d(V T / \Theta) / d z$ is given by

$$
\begin{gathered}
d(V T / \Theta) / d z=u^{2} \frac{d u}{d z} \frac{1}{1+z}, \\
u(z)=\int_{0}^{z} \frac{c}{H\left(z^{\prime}\right)} d z^{\prime} .
\end{gathered}
$$

For the SDSS-II Supernova Survey, the Earth-frame observation time for the 2005-2007 observing seasons are 89, 90 , and 90 days, respectively. The solid angle covered is $\Theta=0.08277 \times 0.98$ sr. As discussed by Dilday et al. (2008), the regions of the difference images that corresponded to the locations of bright stars and objects that showed variability in a previous year, and were thus unlikely to be $\mathrm{SNe}$, were excluded (masked) from the search for SNe. This masking accounts for the factor of $98 \%$ in the computation of the effective solid angle. The value of the volumetric SN Ia rate, as a function of the upper redshift limit for the SN sample, and derived under the assumption of a constant-rate model, is shown in Figure 9. For example, if the upper redshift limit is chosen as $z=0.12$, as in Dilday et al. (2008), then the rate is deter-

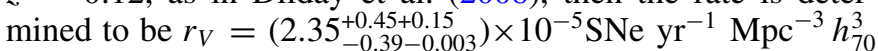
(where $h_{70}=H_{0} /\left(70 \mathrm{~km} \mathrm{~s}^{-1} \mathrm{Mpc}^{-1}\right.$ ), and the quoted uncertainties are statistical and systematic in that order), based on $37 \mathrm{SNe}$ Ia of which 36 are spectroscopically confirmed. This is lower than, although consistent with, the result found by Dilday et al. (2008), $r_{V}=\left(2.93_{-0.17-0.04}^{+0.90+0.71}\right) \times 10^{-5} \mathrm{SNe} \mathrm{yr}^{-1} \mathrm{Mpc}^{-3} h_{70}^{3}$. In relation to the mean low-redshift $\mathrm{SN}$ yield from the three years of the SDSS-II Supernova Survey, the first-year sample presented by Dilday et al. (2008) represented an upward statistical fluctuation. If the upper redshift limit is chosen as $z=0.15$, where the SN sample still has a high degree of completeness, then the SN Ia rate is determined to be

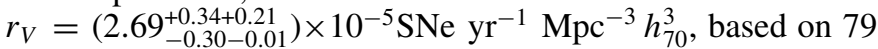
SNe Ia of which 72 are spectroscopically confirmed.

\subsection{SN Ia Rate as a Function of Redshift}

In Figure 10, we plot the volumetric SN Ia rate in running bins of width $\Delta z=0.05$. The rate in each bin is computed by assuming the rate to be constant within the bin, which is a

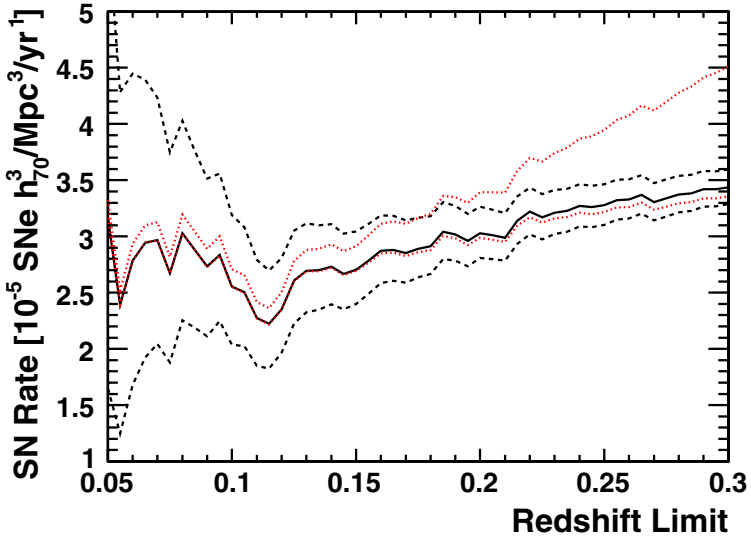

Figure 9. SN Ia rate, assuming a constant-rate model, as a function of upper limit on the redshift range (solid line). The black dashed lines denote the $1 \sigma$ statistical (Poisson) errors. The red dotted lines show estimates of the systematic errors, which include uncertainty in the $\mathrm{SN}$ extinction distribution and estimation of contamination of the SN Ia sample from non-Ia SNe.

(A color version of this figure is available in the online journal.)

good approximation for the small bins considered. The SN rate values are listed in Table 5. It can be seen that the systematic uncertainty in the selection function (due to uncertainty in the extinction distribution) becomes much larger than the statistical uncertainty for $z \gtrsim 0.2$.

As in Dilday et al. (2008), we employ an unbinned maximum likelihood method to fit the $\mathrm{SN}$ rate data to models of the rate as a function of redshift. To review the method, each SN redshift, $z^{i}$, is assumed to be drawn from a probability distribution,

$$
p_{z}^{i} d z=\langle N\rangle^{-1} \Theta T_{\oplus} \epsilon\left(z^{i}\right) r_{V}\left(z^{i}\right) d(V T / \Theta) / d z d z
$$

where $r_{V}(z)$ is the volumetric rate as a function of redshift, $\langle N\rangle$ is the mean number of expected $\mathrm{SNe}$, and the other symbols have been defined in Equation (7). A likelihood function, $L$, can then be formed as a product of the individual probabilities, $p_{z}^{i}$, multiplied by a Poisson distribution of the observed number of $\mathrm{SNe}, N_{\mathrm{SNe}}$,

$$
L=\frac{\langle N\rangle^{N_{\mathrm{SNe}}} e^{-\langle N\rangle}}{\left(N_{\mathrm{SNe}}\right) !} \Pi_{i=1}^{i=N_{\mathrm{SNe}}} p_{z}^{i} .
$$

When the SN redshifts are determined photometrically, the uncertainties in the measurements are significant, and the 


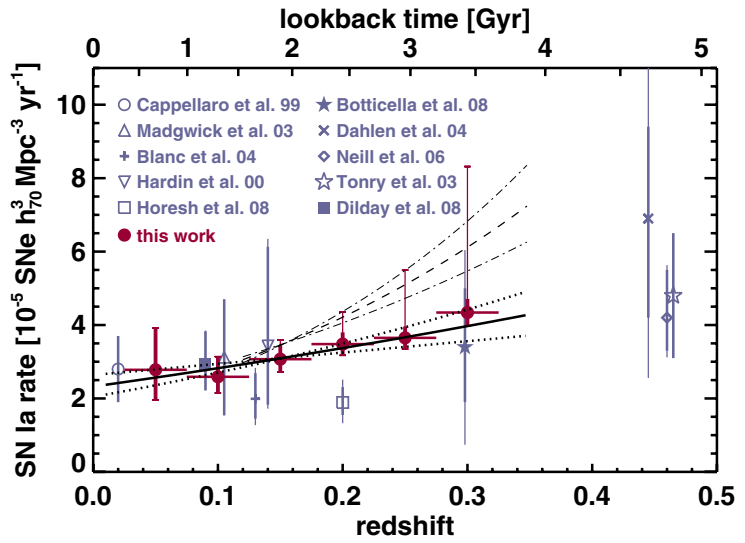

Figure 10. SN Ia rate as a function of redshift for the present work, along with a selection of measurements from the literature. For the measurements presented in this work, the redshift is the median redshift of running bins of size $\Delta z=0.05$, and the $\mathrm{SN}$ rate is computed assuming that the rate is constant in each bin. The thick error bars denote the statistical uncertainty, while the thin error bars denote the systematic uncertainty. The solid line shows the best-fit power-law rate model, and the dotted lines the $1 \sigma$ uncertainty of the best-fit model. The dashed line shows the best-fit power-law rate model (plotted only for $z \geqslant 0.12$ ), assuming a larger mean value of dust extinction (Section 4.1), and the dash-dotted line shows the corresponding $1 \sigma$ uncertainty of the rate model. Some of the SN Ia rate measurements from the literature have been offset in redshift for clarity.

(A color version of this figure is available in the online journal.)

individual probabilities must be modified as

$$
p_{z}^{i}=\langle N\rangle^{-1} \Theta T_{\oplus} \int_{-\infty}^{\infty} \epsilon\left(z^{\prime}\right) r_{V}\left(z^{\prime}\right) d(V T / \Theta) / d z^{\prime} \rho\left(z^{\prime} \mid z^{i}\right) d z^{\prime},
$$

where $\rho\left(z^{\prime} \mid z^{i}\right)$ is the probability that an $\mathrm{SN}$ at redshift $z^{i}$ will have a measured redshift $z^{\prime}$. We assume a Gaussian form for $\rho$,

$$
\rho\left(z^{\prime} \mid z^{i}\right)=\frac{1}{\sqrt{2 \pi} \sigma_{z}} e^{-\left(z^{\prime}-z^{i}\right)^{2} / 2 \sigma_{z}^{2}},
$$

where $\sigma_{z}$ is the uncertainty in the $\mathrm{SN}$ redshift, as determined by the $\mathrm{SN}$ photo- $z$ fits. The parameters of the $\mathrm{SN}$ rate model, $r_{V}$, are then estimated by minimizing the negative log of the likelihood function. This procedure automatically accounts for the bias in the observed SN redshift distribution described in Section 3.3. As discussed in Section 3.4, contamination of the photometric SN sample by non-Ia SNe is accounted for by weighting each photometric SN according to Equation (5).

Using the maximum likelihood formalism described above, we consider an empirical power-law model of the $\mathrm{SN}$ rate as a function of redshift, $r_{V}(z)=A_{p}\left((1+z) /\left(1+z_{0}\right)\right)^{\nu}$. The reference redshift is $z_{0}=0.21$, and the SNe Ia used in the fit are those with $z \leqslant 0.3$. Our minimization and error analysis are performed with the MINUIT $^{29}$ software package, and using the MINOS procedure for asymmetric error estimation. For the power-law rate model, the maximum likelihood estimates of the model

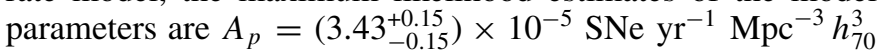
and $v=2.04_{-0.89}^{+0.90}$, with correlation coefficient $\rho=-0.019$. The uncertainties quoted above are the $1 \sigma$ statistical errors, defined as the change in the parameter values such that the log-likelihood function changes by 0.5 . As discussed in Section 4.1, uncertainty in the extinction distribution for SNe Ia has a significant impact on the survey efficiency, as inferred from MC simulations, particularly for $z \gtrsim 0.2$ (Figure 10). Assuming a larger mean

\footnotetext{
29 http://wwwasdoc.web.cern.ch/wwwasdoc/minuit/minmain.html
}

value $\left(\left\langle A_{V}\right\rangle=0.45 \mathrm{mag}\right)$ for the extinction parameter, $A_{V}$, and reevaluating the maximum likelihood estimates for the rate model parameters, results in $A_{p}=\left(4.38_{-0.19}^{+0.20}\right) \times 10^{-5}$ $\mathrm{SNe} \mathrm{yr}^{-1} \mathrm{Mpc}^{-3} h_{70}^{3}$ and $v=4.66_{-0.92}^{+0.93}$, for the parameters of the power-law rate model.

The power-law SN rate models, along with a selection of SN Ia rate measurements from the literature, are shown in Figure 10. SN Ia rate measurements from the SDSS-II Supernova Survey, computed in bins of width $\Delta z=0.05$ (Table 5), are also shown. We emphasize that the binned SDSS-II Supernova Survey points are shown for convenience and ease of comparison, and that the power-law rate models are derived from the unbinned maximum likelihood method (Section 5.2). This plot illustrates the much greater statistical precision of the SDSS-II Supernova Survey in comparison to previous $\mathrm{SN}$ Ia rate measurements in the redshift range $0.1 \lesssim z \lesssim 0.3$. Additionally, the SDSS-II Supernova Survey SN Ia rate measurements in the redshift range $0.1 \lesssim z \lesssim 0.2$ have a relatively small systematic uncertainty, and constrain the SN Ia rate to $\sim 10 \%-20 \%$ total uncertainty. For $z \gtrsim 0.2$, the systematic uncertainty in the SDSS-II Supernova Survey SN Ia rate measurements becomes much greater than the statistical uncertainty. Despite this large systematic uncertainty, the SDSS-II Supernova Survey SN Ia rate measurements have precision comparable to that of the best existing measurements. Finally, we note that the direction of the systematic uncertainty in the SDSS-II Supernova Survey SN Ia rate measurements is toward an increase in the $\mathrm{SN}$ rate. Therefore, the $\mathrm{SN}$ Ia rate measurements presented here provide quite robust lower limits on the $\mathrm{SN}$ Ia rate at $z \lesssim 0.3$.

\section{CONCLUSIONS}

We have measured the volumetric SN Ia rate based on the SDSS-II Supernova Survey using a much larger sample of SNe Ia and a higher redshift limit than was discussed by Dilday et al. (2008). The sample of SNe considered comprises 516 SNe Ia at $z \lesssim 0.3$. The low-redshift portion of the SN Ia sample has a high degree of spectroscopic completeness, while the large redshift range covered enables measurement of the redshift dependence of the SN Ia rate based on the SDSS-II Supernova Survey data alone. Fitting a power-law model to the SN Ia rate, $r_{V}(z)=A_{p}\left((1+z) /\left(1+z_{0}\right)\right)^{v}$, and assuming a distribution for dust extinction as in Kessler et al. (2009), we find $v=2.04_{-0.89}^{+0.90}$. Assuming a larger mean value of dust extinction, we find $v=4.66_{-0.92}^{+0.93}$.

The results presented here represent an order of magnitude improvement in the statistics for SN Ia rate measurements in the same redshift range and solidify the SN Ia rate constraints for $z \lesssim 0.3$. When combined with improved measurements of the cosmic star formation history, the SN Ia rate measurements presented here can be used to place improved constraints on SN Ia progenitor models.

Funding for the SDSS and SDSS-II has been provided by the Alfred P. Sloan Foundation, the Participating Institutions, the National Science Foundation (NSF), the U.S. Department of Energy, the National Aeronautics and Space Administration (NASA), the Japanese Monbukagakusho, the Max Planck Society, and the Higher Education Funding Council for England. The SDSS Web site is http://www.sdss.org/.

The SDSS is managed by the Astrophysical Research Consortium for the Participating Institutions. The Participating Institutions are the American Museum of Natural History, 
Astrophysical Institute Potsdam, University of Basel, University of Cambridge, Case Western Reserve University, University of Chicago, Drexel University, Fermilab, the Institute for Advanced Study, the Japan Participation Group, Johns Hopkins University, the Joint Institute for Nuclear Astrophysics, the Kavli Institute for Particle Astrophysics and Cosmology, the Korean Scientist Group, the Chinese Academy of Sciences (LAMOST), Los Alamos National Laboratory, the Max-PlanckInstitute for Astronomy (MPIA), the Max-Planck-Institute for Astrophysics (MPA), New Mexico State University, Ohio State University, University of Pittsburgh, University of Portsmouth, Princeton University, the United States Naval Observatory, and the University of Washington.

This work is based in part on observations made at the following telescopes. The Hobby-Eberly Telescope (HET) is a joint project of the University of Texas at Austin, the Pennsylvania State University, Stanford University, Ludwig-MaximilliansUniversität München, and Georg-August-Universität Göttingen. The HET is named in honor of its principal benefactors, William P. Hobby and Robert E. Eberly. The Marcario Low-Resolution Spectrograph is named for Mike Marcario of High Lonesome Optics, who fabricated several optical elements for the instrument but died before its completion; it is a joint project of the Hobby-Eberly Telescope partnership and the Instituto de Astronomía de la Universidad Nacional Autónoma de México. The Apache Point Observatory $3.5 \mathrm{~m}$ telescope is owned and operated by the Astrophysical Research Consortium. We thank the observatory director, Suzanne Hawley, and site manager, Bruce Gillespie, for their support of this project. The Subaru Telescope is operated by the National Astronomical Observatory of Japan. The William Herschel Telescope is operated by the Isaac Newton Group, on the island of La Palma in the Spanish Observatorio del Roque de los Muchachos of the Instituto de Astrofisica de Canarias. Based in part on observations made with the Nordic Optical Telescope, operated on the island of La Palma jointly by Denmark, Finland, Iceland, Norway, and Sweden, in the Spanish Observatorio del Roque de los Muchachos of the Instituto de Astrofisica de Canarias. Kitt Peak National Observatory, National Optical Astronomy Observatory, is operated by the Association of Universities for Research in Astronomy (AURA), Inc., under cooperative agreement with the NSF. The W. M. Keck Observatory is operated as a scientific partnership among the California Institute of Technology, the University of California, and NASA; it was made possible by the generous financial support of the W. M. Keck Foundation. Based partially on observations made with the Italian Telescopio Nazionale Galileo (TNG) operated on the island of La Palma by the Fundación Galileo Galilei of the INAF (Istituto Nazionale di Astrofisica) at the Spanish Observatorio del Roque de los Muchachos of the Instituto de Astrofísica de Canarias.

This work was supported in part by the Kavli Institute for Cosmological Physics at the University of Chicago through grants NSF PHY-0114422 and NSF PHY-0551142, and by an endowment from the Kavli Foundation and its founder Fred Kavli. This work was also partially supported by the US Department of Energy through grants DE-FG02-08ER41562 to Rutgers University (PI: S.W.J.) and DE-FG02-08ER41563 to U. C. Berkeley (PI: A.V.F.), as well as by NSF grants AST-0607485 and AST-0908886 (PI: A.V.F.).

\section{REFERENCES}

Alard, C., \& Lupton, R. H. 1998, ApJ, 503, 325

Aldering, G., et al. 2006, ApJ, 650, 510
Astier, P., et al. 2006, A\&A, 447, 31

Barris, B. J., \& Tonry, J. L. 2006, ApJ, 637, 427

Bazin, G., et al. 2009, A\&A, 499, 653

Blanc, G., et al. 2004, A\&A, 423, 881

Botticella, M. T., et al. 2008, A\&A, 479, 49

Cappellaro, E., Evans, R., \& Turatto, M. 1999, A\&A, 351, 459

Dahlen, T., Strolger, L.-G., \& Riess, A. G. 2008, ApJ, 681, 462

Dahlen, T., et al. 2004, ApJ, 613, 189

D'Andrea, C. B., et al. 2010, ApJ, 708, 661

Dilday, B., et al. 2008, ApJ, 682, 262

Dilday, B., et al. 2010, arXiv:1003.1521

Filippenko, A. V. 1997, ARA\&A, 35, 309

Filippenko, A. V. 2005, in White Dwarfs: Cosmological and Galactic Probes, Astrophys. and Space Science Lib. Vol. 332, ed. E. M. Sion, S. Vennes, \& H. L. Shipman (Dordrecht: Springer), 97

Förster, F., Wolf, C., Podsiadlowski, P., \& Han, Z. 2006, MNRAS, 368, 1893

Freedman, W. L., et al. 2009, ApJ, 704, 1036

Frieman, J. A., et al. 2008, AJ, 135, 338

Fukugita, M., Ichikawa, T., Gunn, J. E., Doi, M., Shimasaku, K., \& Schneider, D. P. 1996, AJ, 111, 1748

Gal-Yam, A., Cenko, S. B., Fox, D. B., Leonard, D. C., Moon, D.-S., Sand, D. J., \& Soderberg, A. M. 2007, in AIP Conf. Proc. 924, The Multicolored Landscape of Compact Objects and their Explosive Origins, ed. T. Di Salvo et al. (Melville, NY: AIP), 297

Gal-Yam, A., Cenko, S. B., Fox, D. W., Leonard, D. C., Moon, D.-S., Sand, D. J., \& Soderberg, A. M. 2005, in ASP Conf. Ser. 342, 1604-2004: Supernovae as Cosmological Lighthouses, ed. M. Turatto et al. (San Francisco, CA: ASP), 305

Greggio, L. 2005, A\&A, 441, 1055

Gunn, J. E., et al. 1998, AJ, 116, 3040

Gunn, J. E., et al. 2006, AJ, 131, 2332

Hardin, D., et al. 2000, A\&A, 362, 419

Hicken, M., Wood-Vasey, W. M., Blondin, S., Challis, P., Jha, S., Kelly, P. L., Rest, A., \& Kirshner, R. P. 2009, ApJ, 700, 1097

Hogg, D. W., Finkbeiner, D. P., Schlegel, D. J., \& Gunn, J. E. 2001, AJ, 122, 2129

Holtzman, J. A., et al. 2008, AJ, 136, 2306

Horesh, A., Poznanski, D., Ofek, E. O., \& Maoz, D. 2008, MNRAS, 389, 1871

Ivezić, Ž., et al. 2004, Astron. Nachr., 325, 583

Jha, S., Riess, A. G., \& Kirshner, R. P. 2007, ApJ, 659, 122

Kessler, R., et al. 2009, ApJS, 185, 32

Kuznetsova, N. V., \& Connolly, B. M. 2007, ApJ, 659, 530

Kuznetsova, N., et al. 2008, ApJ, 673, 981

$\mathrm{Li}, \mathrm{W}$., et al. 2010a, submitted

Li, W., et al. 2010b, submitted

Lupton, R. H., Gunn, J. E., \& Szalay, A. S. 1999, AJ, 118, 1406

Madgwick, D. S., Hewett, P. C., Mortlock, D. J., \& Wang, L. 2003, ApJ, 599, L33

Neill, J. D., et al. 2006, AJ, 132, 1126

Oyaizu, H., Lima, M., Cunha, C. E., Lin, H., \& Frieman, J. 2008, ApJ, 689, 709

Pain, R., et al. 2002, ApJ, 577, 120

Perlmutter, S., et al. 1999, ApJ, 517, 565

Phillips, M. M. 1993, ApJ, 413, L105

Poznanski, D., Maoz, D., \& Gal-Yam, A. 2007a, AJ, 134, 1285

Poznanski, D., et al. 2007b, MNRAS, 382, 1169

Prieto, J. L., et al. 2007, arXiv:0706.4088

Pskovskii, Yu. P. 1977, Sov. Astron., 21, 675

Richardson, D., Branch, D., \& Baron, E. 2006, AJ, 131, 2233

Richardson, D., Branch, D., Casebeer, D., Millard, J., Thomas, R. C., \& Baron, E. 2002, AJ, 123, 745

Riess, A. G., et al. 1998, AJ, 116, 1009

Riess, A. G., et al. 2004, ApJ, 607, 665

Riess, A. G., et al. 2007, ApJ, 659, 98

Riess, A. G., et al. 2009, ApJS, 183, 109

Sako, M., et al. 2008, AJ, 135, 348

Schechter, P. L., Mateo, M., \& Saha, A. 1993, PASP, 105, 1342

Smith, J. A., et al. 2002, AJ, 123, 2121

Stoughton, C., et al. 2002, AJ, 123, 485

Sullivan, M., et al. 2006a, AJ, 131, 960

Sullivan, M., et al. 2006b, ApJ, 648, 868

Tonry, J. L., et al. 2003, ApJ, 594, 1

Tucker, D. L., et al. 2006, Astron. Nachr., 327, 821

Wood-Vasey, W. M., et al. 2007, ApJ, 666, 694

York, D. G., et al. 2000, AJ, 120, 1579

Zheng, C., et al. 2008, AJ, 135, 1766

Zwicky, F. 1938, ApJ, 88, 529 